\title{
Energy-Efficient PCF Operation of IEEE 802.11a Wireless LAN
}

\author{
Daji Qiao $^{+}$ \\ ${ }^{+}$Real-Time Computing Laboratory \\ The University of Michigan \\ Ann Arbor, MI 48109, USA \\ \{dqiao,kgshin\}@eecs.umich.edu
}

\author{
Amjad Soomro* Kang G. Shin ${ }^{+}$ \\ *Wireless Communications and Networking \\ Philips Research USA \\ Briarcliff Manor, NY 10510, USA \\ \{sunghyun.choi,amjad.soomro\}@philips.com
}

\begin{abstract}
In this paper, we demonstrate the energy-efficient Point Coordination Function (PCF) operation of IEEE 802.11a wireless LAN (WLAN) via both transmit power control (TPC) and physical layer (PHY) rate adaptation. First, we derive the energy-consumption performance analytically for uplink data transmissions under the PCF. From the evaluation results, we observe that significant energy savings can be achieved by combining TPC with adaptive PHY rate selection. A key requirement for a transmitter to select the most energy-efficient combination of transmit power level and PHY rate is the knowledge of the path loss between the receiver and itself. We present a novel scheme for accurate path loss estimation in 802.11 WLANs. Results and conclusions presented in this paper can serve as a valuable guidance or reference for the design of future $5 \mathrm{GHz}$ 802.11 WLAN systems.
\end{abstract}

\section{INTRODUCTION}

For wide-area cellular systems, such as IS-95 CodeDivision Multiple Access (CDMA) and the third generation (3G) Wide-band CDMA (W-CDMA), transmit power control (TPC) is critically important in order to (1) ameliorate the near-far problem, specifically, for CDMA uplink systems; (2) minimize the interference to/from other cells, i.e., co-channel interference; and (3) improve the system performance on fading channels by compensating fading dips [1]. For wireless local area networks (WLANs), which are mainly used in indoor home, office, and public access environments, TPC has not attracted enough attention as it was not considered as critical to success as in CDMA systems. However, since many WLAN devices such as laptops and palmtops are battery-powered, and extending the operation time of such devices is always desirable, applying TPC in WLAN systems in order to save the battery energy can be naturally an attractive idea. Moreover, in the multi-cell WLAN systems often found in office and public access environments, reducing the co-channel interference via TPC could be quite beneficial as well since it results in better error performance in a given area. In this paper, we demonstrate the energy-efficient data transmission in IEEE 802.11a WLANs by combining TPC with physical layer (PHY) rate adaptation.

The work reported in this paper was supported in part by AFOSR under Grant No. F49620-00-1-0327.

\section{A. Problem Statement}

The IEEE 802.11 standard [2] specifies two different medium access control (MAC) mechanisms in WLANs: the basic access mechanism, called the Distributed Coordination Function (DCF), based on Carrier-Sense Multiple Access with Collision Avoidance (CSMA/CA), and a centrally-controlled access mechanism, called the Point Coordination Function (PCF), based on polling. The IEEE 802.11a PHY [3] is the new high-speed PHY developed to operate IEEE 802.11 in the $5 \mathrm{GHz}$ Unlicensed National Information Infrastructure (UNII) band, which provides 8 PHY modes with data transmission rates ranging from $6 \mathrm{Mbps}$ up to $54 \mathrm{Mbps}$.

In [4], we derived the goodput performance analytically for peer-to-peer communication in an IEEE 802.11a WLAN under the DCF, and proposed a link adaptation scheme to achieve goodput enhancement via both dynamic fragmentation and adaptive PHY mode (or equivalently, transmission rate) selection. In this paper, we address another important problem in the WLAN environment: how to minimize the energy consumption for data transmissions under the PCF? Obviously, in order to deliver a data frame, the higher the PHY rate, the shorter the transmission time and the less energy consumed in one transmission attempt, but more likely the transmission will fail, thus engendering re-transmissions. So, there is an inherent trade-off, and our idea is to combine TPC with adaptive PHY mode selection, so that the proper PHY rate as well as the best transmit power level can be adaptively selected to combat the path loss variation, thus delivering data with the minimum energy consumption.

It may seem reasonable to apply the similar idea under the DCF as well. However, as described in [5], by allowing wireless stations to transmit at different power levels under the DCF, the number of hidden terminals is likely to increase, which, in turn, results in more collisions and retransmissions due to the very nature of DCF's contentionbased access mechanism, and hence, more energy is consumed eventually. Therefore, the energy saving hoped for with power reduction is unlikely to be achieved, thus making TPC undesirable for the DCF operation of an IEEE 802.11 WLAN. On the other hand, there is no "hidden node" problem under the PCF, since access to the wireless medium is centrally-controlled by the Point Coordinator (PC), or equivalently, the Access Point 
(AP). Besides, the PC is normally located at a fixed position and connected to the power line, and therefore, energy consumption at the PC for downlink (PC-to-station) data transmissions is usually not a critical issue. We are more concerned about energy savings by battery-operated wireless stations for uplink (station-to-PC) data transmissions under the PCF.

One may think that the energy-efficiency optimization does not constitute the ultimate goal for the PCF operation since the PCF is typically used for isochronous real-time services. While this is a valid observation as real-time services require a timely delivery of traffic with less error, which may not result in energy-efficient operations, it should be also noted that the polling-based PCF could be used for non-real-time services as well. For example, the PCF is known to achieve a higher maximum throughput than the contention-based DCF [6], and hence one may want to use the PCF instead of the DCF in order to maximize the system throughput for data traffic. Our paper basically shows that one may also prefer the PCF with the proposed TPC to the DCF without TPC in order to minimize the energy consumption. We also consider a more typical PCF application in this paper, i.e., a streaming-like service that requires a sustained goodput level, and demonstrate the energy-efficient PCF operation with the minimum goodput constraint.

A WLAN device operates in one of the following modes: transmit mode, receive mode, or sleeping mode. Transmit mode results in the highest energy consumption, while sleeping mode consumes the least amount of energy. The TPC mechanism will be included in the upcoming IEEE $802.11 \mathrm{~h}$ standard [7], which is an extension to the current 802.11 MAC and 802.11a PHY, and will allow a WLAN device to use one of several available power levels in transmit mode. The results and conclusions presented in this paper should be a valuable guidance or reference for the future $802.11 \mathrm{a} / \mathrm{h}$ WLAN system design.

\section{B. Related Work}

In recent years, several power-management policies have been proposed to force a WLAN device to sleep adaptively at appropriate moments to save battery energy. In [8], the authors used the Time-Indexed Semi-Markov Decision Process (TISMDP) model to derive the optimal policy for dynamic power management in portable systems. In [9], several application-specific policies were given to put an idle WLAN device into sleeping mode. However, both papers assumed a fixed transmit power level. Since TPC determines the best transmit power level to use in transmit mode, it is complementary to these power-management policies, which address how to switch between transmit/receive and sleeping modes.

The authors of [10] presented a scheme where the most battery energy-efficient combination of Forward Error Correction (FEC) code and Automatic Re-transmission reQuest (ARQ) protocol is chosen and adapted over time for data transmissions without, however, considering TPC.

\section{Organization}

The rest of this paper is organized as follows. Section II introduces the PCF of IEEE 802.11 MAC as well as the IEEE 802.11a PHY. The error probability analysis and the energy consumption analysis are presented in Section III and IV, respectively. Section V presents the evaluation results, and the implementation issues are discussed in Section VI. Finally, this paper concludes with Section VII.

\section{System OVERVIEW}

\section{A. PCF of IEEE 802.11 MAC}

The centrally-coordinated access mechanism of the IEEE 802.11 MAC, called the PCF, adopts a poll-and-response protocol to control the access to the shared wireless medium and eliminate contention among wireless stations. It makes use of the priority inter-frame space (PIFS) to seize and maintain control of the medium. The period during which the PCF is operated is called the contention-free period (CFP). ${ }^{1}$ Once the PC has control of the medium, it may start transmitting downlink traffic to stations. Alternatively, the PC can also send contention-free poll (CF-Poll) frames to those stations that have requested contention-free services for their uplink traffic. During a CFP, a wireless station can only transmit after being polled by the PC. If a polled station has uplink traffic to send, it may transmit one frame for each CF-Poll received. Otherwise, it will respond with a NULL frame, which is a data frame without any payload. Besides, in order to utilize the medium more efficiently during the CFP, it is possible to piggyback both the acknowledgment (CF-Ack) and the CF-Poll onto data frames.

During the CFP, the PC sends a frame to a wireless station and expects the reply frame, either a CF-Ack or a data frame or a NULL frame in response to a CF-Poll, within a short interframe space (SIFS) that is shorter than PIFS. Consider an example of uplink data frame transmission. The PC first sends a CF-Poll to the wireless station and waits for an uplink data frame. As shown in Fig. 1, if a data frame is received correctly within SIFS time, the PC will send a CF-Ack+CF-Poll frame that allows the next uplink data frame transmission. If a data frame is received in error, determined by an incorrect Frame Check Sequence (FCS), or equivalently, an incorrect Cyclic Redundancy Check (CRC), the PC will send a CF-Poll asking for the re-transmission, as shown in Fig. 2. However, if no reply frame is received within a SIFS interval possibly due to an erroneous reception of the preceding CF-Poll frame by the polled station, the PC will reclaim the medium and send its next CF-Poll after a PIFS interval from the end of the previous CF-Poll frame, as shown in Fig. 3. In this case, the PC will

\footnotetext{
${ }^{1}$ In an IEEE 802.11 WLAN, a Contention-Free Period (CFP) and a Contention Period (CP) alternate over time periodically, where the centrallycoordinated PCF is used during a CFP, and the contention-based DCF is used during a $\mathrm{CP}$.
} 
not be confused with the scenario where the polled station has nothing to transmit, because a NULL frame is expected under that circumstance. Therefore, the PC may choose to re-poll the same station instead of skipping to poll the next station on its polling list. Note that, in these figures, the blocks labeled with "CF-Ack $(i)$ ", "CF-Poll $(i)$ ", and "Frame $(i)$ " represent the acknowledgment to, the contention-free poll to, and the uplink frame transmission from station $i$, respectively, and a crossed block represents an erroneous reception of the corresponding frame.

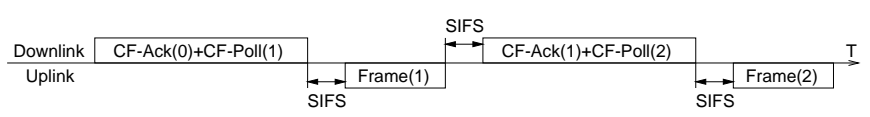

Fig. 1. Timing of successful uplink frame transmissions under the PCF

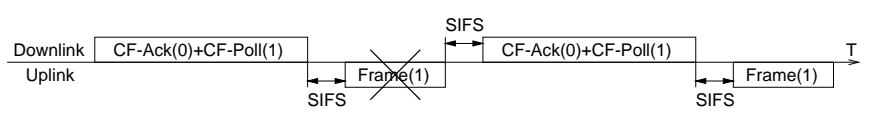

Fig. 2. CF-poll re-transmission due to an erroneous data frame reception

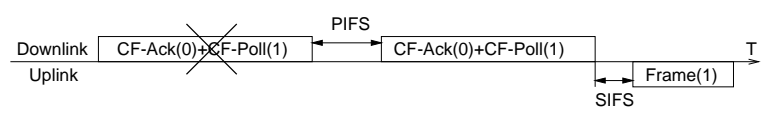

Fig. 3. CF-poll re-transmission due to CF-Poll failure

\section{B. IEEE 802.11a PHY}

The PHY is the interface between the MAC and the wireless medium, which transmits and receives data frames over the shared wireless medium. The frame exchange between MAC and PHY is under the control of the Physical Layer Convergence Procedure (PLCP) sublayer.

The IEEE 802.11a PHY is based on Orthogonal Frequency Division Modulation (OFDM), and provides 8 PHY modes with different modulation schemes and different convolutional coding rates at the $5 \mathrm{GHz}$ U-NII band. As listed in Table I, the OFDM system provides a WLAN with capabilities of communicating at 6 to $54 \mathrm{Mbps}$. Forward error correction is performed by bit interleaving and rate- $1 / 2$ convolutional coding. The higher code rates of $2 / 3$ and $3 / 4$ are obtained by puncturing the original rate- $1 / 2$ code.

\section{ERror PROBABILITy ANALYSIS}

In this paper, we assume that the noise over the wireless medium is white Gaussian with spectral density $N_{0} / 2$, and use the same error probability analysis as in [4]. For completeness, we briefly describe the analysis.
TABLE I

EIGHT PHY MODES OF IEEE 802.11A OFDM PHY

\begin{tabular}{|c|c|c|c|c|}
\hline Mode & Modulation & Code Rate & Data Rate & BpS \\
\hline \hline 1 & BPSK & $1 / 2$ & $6 \mathrm{Mbps}$ & 3 \\
\hline 2 & BPSK & $3 / 4$ & $9 \mathrm{Mbps}$ & 4.5 \\
\hline 3 & QPSK & $1 / 2$ & $12 \mathrm{Mbps}$ & 6 \\
\hline 4 & QPSK & $3 / 4$ & $18 \mathrm{Mbps}$ & 9 \\
\hline 5 & $16-Q A M$ & $1 / 2$ & $24 \mathrm{Mbps}$ & 12 \\
\hline 6 & $16-Q A M$ & $3 / 4$ & $36 \mathrm{Mbps}$ & 18 \\
\hline 7 & 64-QAM & $2 / 3$ & $48 \mathrm{Mbps}$ & 24 \\
\hline 8 & 64-QAM & $3 / 4$ & $54 \mathrm{Mbps}$ & 27 \\
\hline
\end{tabular}

\section{A. Bit Error Probability}

The symbol error probability for an $M$-ary Quadrature Amplitude Modulation (QAM) [11] with $M=4,16,64$ can be calculated by

$$
P_{M}=1-\left(1-P_{\sqrt{M}}\right)^{2},
$$

where

$$
P_{\sqrt{M}}=2 \cdot\left(1-\frac{1}{\sqrt{M}}\right) \cdot Q\left(\sqrt{\frac{3}{M-1} \cdot \frac{E_{a v}}{N_{0}}}\right)
$$

is the symbol error probability for the $\sqrt{M}$-ary Pulse Amplitude Modulation (PAM) with the average signal-to-noise ratio (SNR) per symbol, $E_{a v} / N_{o}$. The Q-function is defined as

$$
Q(x)=\int_{x}^{\infty} \frac{1}{\sqrt{2 \pi}} e^{-y^{2} / 2} d y
$$

With a Gray coding, the bit error probability for an $M$-ary QAM modulation can be approximated by

$$
P_{b}^{(M)} \approx \frac{1}{\log _{2} M} \cdot P_{M}
$$

For Binary Phase Shift Keying (BPSK) modulation, the bit error probability is the same as the symbol error probability, which is given by

$$
P_{b}^{(2)}=P_{2}=Q\left(\sqrt{\frac{2 E_{a v}}{N_{0}}}\right) .
$$

The SNR (in $\mathrm{dB}$ ) used above actually equals the output power level (in $\mathrm{dBm}$ ) at the transmitter minus the path loss (in $\mathrm{dB}$ ) and the white Gaussian noise level (in $\mathrm{dBm}$ ) observed at the receiver. Therefore, the error performance of a modulation scheme varies for different transmit power levels and different path loss conditions.

\section{B. Packet Error Probability}

In [12], an upper bound was given on the packet error probability, under the assumption of binary convolutional coding 
and hard-decision Viterbi decoding with independent errors at the channel input. For an $L$-octet long packet to be transmitted using PHY mode $m(1 \leq m \leq 8)$, this bound is

$$
P_{e}^{m}(L) \leq 1-\left(1-P_{u}^{m}\right)^{8 L},
$$

where the union bound $P_{u}^{m}$ of the first-event error probability is given by

$$
P_{u}^{m}=\sum_{d=d_{\text {free }}}^{\infty} a_{d} \cdot P_{d}
$$

where $d_{f r e e}$ is the free distance of the convolutional code selected in PHY mode $m, a_{d}$ is the total number of error events of weight $d$, and $P_{d}$ is the probability that an incorrect path at distance $d$ from the correct path being chosen by the Viterbi decoder. When hard decision decoding is applied, $P_{d}$ is given by

$$
P_{d}= \begin{cases}\sum_{k=(d+1) / 2}^{d}\left(\begin{array}{l}
d \\
k
\end{array}\right) \rho^{k}(1-\rho)^{d-k}, & \text { if } d \text { is odd, } \\
\frac{1}{2}\left(\begin{array}{c}
d \\
d / 2
\end{array}\right) \rho^{d / 2}(1-\rho)^{d / 2} & \\
+\sum_{k=d / 2+1}^{d}\left(\begin{array}{l}
d \\
k
\end{array}\right) \rho^{k}(1-\rho)^{d-k}, & \text { if } d \text { is even, }\end{cases}
$$

where $\rho$ is the bit error probability for the modulation scheme selected in PHY mode $m$, and is given by Eq. (4) or (5). The value of $a_{d}$ can be obtained either from the transfer function or by a numerical search [13].

\section{ENERGy CONSUMPTION ANALYSIS}

In this section, we analyze the average energy consumed by a wireless station to deliver an $L$-octet long data frame successfully (after potential re-transmissions) to the PC under the PCF.

\section{A. Assumptions}

The objective is to analyze the energy consumption of an 802.11a WLAN device when it is actively transmitting, receiving, or sensing the channel, i.e., when it is not in sleeping mode. The energy consumption is measured in Joule per delivered data bit. Since we do not have access to the energyconsumption characteristics of the 802.11a-compliant products currently available in the market, we make some assumptions for our analysis based on the power characteristics of two 802.11b-compliant WLAN devices, the Agere ORiNOCO (or formerly Lucent WaveLAN) card [14] and the Intersil Prism II card [15][16], which are listed in Tables II and III, respectively. The IEEE 802.11b PHY [17] is another high-speed physical layer extension to IEEE 802.11, which provides data rates up to $11 \mathrm{Mbps}$ in the $2.4 \mathrm{GHz}$ band.

Note that the power consumption is different for the receive and transmit modes of both cards, because different circuits are used in different modes. A simplified block diagram for a WLAN card is shown in Fig. 4. The RF power amplifier (PA)
TABLE II

POWER CHARACTERISTICS OF AGERE ORINOCO CARD

\begin{tabular}{|l|l|}
\hline Receive Mode & $180 \mathrm{~mA}$ \\
\hline Transmit Mode & $280 \mathrm{~mA}$ \\
\hline Power Supply & $5 \mathrm{~V}$ \\
\hline \hline Nominal Output Power & $15 \mathrm{dBm}$ \\
\hline
\end{tabular}

TABLE III

POWER CHARACTERISTICS OF INTERSIL PRISM II CARD

\begin{tabular}{|l|l|}
\hline Continuous Receive Mode & $185 \mathrm{~mA}$ \\
\hline Continuous Transmit Mode & $300 \mathrm{~mA}$ \\
\hline Power Amplifier Supply Current & $180 \mathrm{~mA}$ \\
\hline Power Supply & $3.3 \mathrm{~V}$ \\
\hline \hline Nominal Output Power & $18 \mathrm{dBm}$ \\
\hline
\end{tabular}

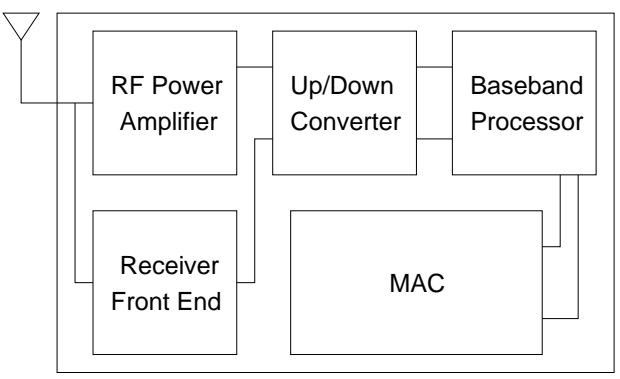

Fig. 4. Simplified block diagram of a WLAN card

is active in transmit mode only, while the receiver front end (e.g., the low noise amplifier in an Intersil Prism II card) is active only in receive mode.

The power conversion efficiency $(\eta)$ of a PA is defined as the ratio of the signal power emitted from the antenna, or the output (transmit) power level $\left(\mathcal{P}_{\text {out }}\right)$, to the total power consumed by the PA $\left(\mathcal{P}_{p a}\right)$. Basically, $\eta$ is a function of $\mathcal{P}_{\text {out }}$, and a PA presents the following non-linearity characteristic: it achieves very high efficiency at high output power levels, but the efficiency drops flat at low power levels. The E-P (efficiency vs. output power level) curve varies for different PA designs. Based on the E-P curves given in [18] and [19], we assume exponential E-P curves (see Fig. 7) for the $5 \mathrm{GHz}$ power amplifiers to be used in the 802.11a-compliant WLAN devices to simplify our analysis. Since we are only interested in how to save energy by using PHY rate adaptation with TPC, not the exact amount of energy savings, this assumption has little impact on the results to be presented in Section V.

Let $\mathcal{P}_{\text {rec }}$ denote the power consumption of the receiver front end. In general, $\mathcal{P}_{r e c}$ is lower than $\mathcal{P}_{p a}$, and the difference becomes significant when the output power level is high. Converter, baseband processor, and MAC are considered to be the common components of both receive and transmit circuits, and they are assumed to consume the same amount of power 
$\left(\mathcal{P}_{\text {com }}\right)$ in both receive and transmit modes. Let $\mathcal{P}_{r_{-} \text {mode }}$ and $\mathcal{P}_{t \_m o d e}$ be the total power consumption in receive and transmit modes, respectively. Then, we have:

$$
\left\{\begin{array}{l}
\mathcal{P}_{r_{-} \text {mode }}=\mathcal{P}_{\text {com }}+\mathcal{P}_{\text {rec }}, \\
\mathcal{P}_{t_{-} \text {mode }}\left(\mathcal{P}_{\text {out }}\right)=\mathcal{P}_{\text {com }}+\mathcal{P}_{\text {pa }}=\mathcal{P}_{\text {com }}+\frac{\mathcal{P}_{\text {out }}}{\eta\left(\mathcal{P}_{\text {out }}\right)}
\end{array}\right.
$$

Furthermore, we assume that there is no retry limit for each frame, i.e., each frame will be (re)transmitted until it is successfully delivered, and the air propagation delays are negligible. Finally, we assume that the $\mathrm{CF}-\mathrm{Ack}+\mathrm{CF}-\mathrm{Poll}$ frame is transmitted at the same rate as the data frame which it is acknowledging.

\section{B. MAC/PHY Layer Overheads}

As shown in Fig. 5, in the IEEE 802.11 MAC, each MAC data frame, or MAC Protocol Data Unit (MPDU), consists of the following components: MAC header, variable-length information frame body, and frame check sequence (FCS). The MAC overheads due to the MAC header and the FCS are 28 octets in total. A CF-Ack+CF-Poll frame uses the same frame format as a data frame, but with zero frame body and different values in the subtype subfield of the frame control field.

\begin{tabular}{|c|c|c|c|c|c|c|c|} 
octets: 2 & 2 & 6 & 6 & 2304 & 4 \\
\hline $\begin{array}{r}\text { Frame } \\
\text { Control }\end{array}$ & $\begin{array}{c}\text { Duration/ } \\
\text { ID }\end{array}$ & Address 1 & Address 2 & Address 3 & $\begin{array}{c}\text { Sequence } \\
\text { Control }\end{array}$ & Frame Body & FCS \\
\hline
\end{tabular}

Fig. 5. Frame format of a data frame MPDU

During the transmission, a PLCP preamble and a PLCP header are added to an MPDU to create a PLCP Protocol Data Unit (PPDU). The PPDU format of the IEEE 802.11a PHY is shown in Fig. 6, which includes PLCP preamble, PLCP header, MPDU (conveyed from MAC), tail bits, and pad bits, if necessary. The PLCP header except the SERVICE field, with the duration of $t P L C P \_S I G$, constitutes a single OFDM symbol, which is transmitted with BPSK modulation and the rate-1/2 convolutional coding. Each OFDM symbol interval, denoted by $t$ Symbol, is $4 \mu s$. The 16-bit SERVICE field of the PLCP header and the MPDU (along with 6 tail bits and pad bits), represented by DATA, are transmitted at the data rate specified in the RATE field. Table IV lists the related characteristics for the IEEE 802.11a PHY.

Based on the above analysis, for $L$-octet long information to be transmitted over the IEEE 802.11a physical layer using PHY mode $m$, the transmission duration is

$$
\begin{aligned}
T_{\text {data }}^{m}(L)= & t P L C P \text { Preamble }+ \text { tPLCP_SIG } \\
& +\left\lceil\frac{30.75+L}{B p S(m)}\right\rceil \cdot t \text { Symbol } \\
= & 20 \mu s+\left\lceil\frac{30.75+L}{B p S(m)}\right\rceil \cdot 4 \mu s .
\end{aligned}
$$

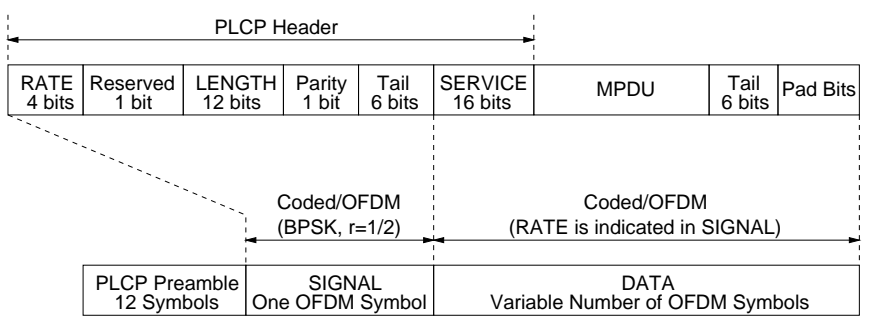

Fig. 6. PPDU frame format of IEEE 802.11a OFDM PHY

TABLE IV

IEEE 802.11A OFDM PHY CHARACTERISTICS

\begin{tabular}{|l|l|l|}
\hline Characteristics & Value & Comments \\
\hline \hline aSlotTime & $9 \mu s$ & Slot time \\
\hline aSIFSTime & $16 \mu \mathrm{s}$ & SIFS time \\
\hline aPIFSTime & $25 \mu \mathrm{s}$ & PIFS = SIFS + Slot \\
\hline tPLCPPreamble & $16 \mu \mathrm{s}$ & PLCP preamble duration \\
\hline tPLCP_SIG & $4 \mu \mathrm{s}$ & PLCP SIGNAL field duration \\
\hline$t$ Symbol & $4 \mu \mathrm{s}$ & OFDM symbol interval \\
\hline
\end{tabular}

Note that the Bytes-per-Symbol information for PHY mode $m, B p S(m)$, is given in Table I. Similarly, the transmission duration for a CF-Ack+CF-Poll frame using PHY mode $m$ is

$$
T_{a c k / p o l l}^{m}=T_{d a t a}^{m}(0) .
$$

\section{Energy Consumption Analysis}

Assume that $L$-octet long information is transmitted using PHY mode $m$ with output power level $\mathcal{P}_{\text {out }}$. Then, the probability of a successful transmission can be calculated by

$$
P_{\text {good_cycle }}^{m}(L)=\left(1-P_{e, \text { ack } / \text { poll }}^{m}\right) \cdot\left(1-P_{e, \text { data }}^{m}(L)\right),
$$

where $P_{e, a c k / p o l l}^{m}$ and $P_{e, \text { data }}^{m}(L)$ are the CF-Ack+CF-Poll transmission error probability and the data transmission error probability, respectively, and can be calculated by

$$
P_{e, a c k / p o l l}^{m}=1-\left(1-P_{e}^{1}(3)\right) \cdot\left(1-P_{e}^{m}(30.75)\right)
$$

and

$$
P_{e, d a t a}^{m}(L)=1-\left(1-P_{e}^{1}(3)\right) \cdot\left(1-P_{e}^{m}(30.75+L)\right) .
$$

Here, $P_{e}^{1}(3)$ is the error probability of the PLCP SIGNAL field, because it is 24-bit long and always transmitted with PHY mode 1. $P_{e}^{1}(\cdot)$ and $P_{e}^{m}(\cdot)$ are calculated by Eq. (6).

Based on the analysis in Section II-A, the expected total energy consumption, $\mathcal{E}_{\text {data }}$, for an uplink data frame delivery can be calculated by Eq. (15) (on next page), where $\overline{\mathcal{E}}_{r e-x m i t}$ is the expected energy consumed for each re-transmission. $\overline{\mathcal{E}}_{\text {re-xmit }}, T_{\text {data }}^{m}(L), T_{\text {ack } / \text { poll }}^{m}$, and $\mathcal{P}_{r_{-} \text {mode }}, \mathcal{P}_{t \_m o d e}$ are given by Eqs. (16) (on next page), (10), (11), and (9), respectively, and

$$
P[n=i]=\left[1-P_{\text {good_cycle }}^{m}(L)\right]^{i} \cdot P_{\text {good_cycle }}^{m}(L)
$$




$$
\begin{aligned}
& \mathcal{E}_{\text {data }}=\sum_{i=1}^{\infty} P[n=i] \cdot i \cdot \overline{\mathcal{E}}_{\text {re-xmit }}+T_{\text {data }}^{m}(L) \cdot \mathcal{P}_{t_{-} \text {mode }}\left(\mathcal{P}_{\text {out }}\right)+\left(T_{\text {ack } / \text { oll }}^{m}+2 \cdot a \text { SIFSTime }\right) \cdot \mathcal{P}_{r_{-} \text {mode }} \\
& \overline{\mathcal{E}}_{\text {re-xmit }}=\frac{\left(1-P_{e, a c k / \text { poll }}^{m}\right) \cdot P_{e, \text { data }}^{m}(L)}{1-P_{\text {good_cycle }}^{m}(L)} \cdot\left[T_{\text {data }}^{m}(L) \cdot \mathcal{P}_{t \_ \text {mode }}\left(\mathcal{P}_{\text {out }}\right)+\left(T_{\text {ack } / \text { poll }}^{m}+2 \cdot \text { aSIFSTime }\right) \cdot \mathcal{P}_{r_{-} \text {mode }}\right] \\
& +\frac{P_{e, a c k / p o l l}^{m}}{1-P_{\text {good_cycle }}^{m}(L)} \cdot\left[\left(T_{a c k / p o l l}^{m}+a P I F S T i m e\right) \cdot \mathcal{P}_{r_{-} \text {mode }}\right] .
\end{aligned}
$$

$$
\begin{aligned}
\mathcal{D}_{\text {data }}= & \sum_{i=1}^{\infty} P[n=i] \cdot i \cdot\left[\frac{\left(1-P_{e, \text { ack } / \text { poll }}^{m}\right) \cdot P_{e, \text { data }}^{m}(L)}{1-P_{\text {good_cycle }}^{m}(L)} \cdot\left(T_{\text {data }}^{m}(L)+T_{\text {ack } / \text { poll }}^{m}+2 \cdot \text { aSIFSTime }\right)\right. \\
& \left.+\frac{P_{e, \text { ack } / \text { poll }}^{m}}{1-P_{\text {good_cycle }}^{m}(L)} \cdot\left(T_{\text {ack } / \text { poll }}^{m}+\text { aPIFSTime }\right)\right]+T_{\text {data }}^{m}(L)+T_{\text {ack } / \text { poll }}^{m}+2 \cdot \text { aSIFSTime. }
\end{aligned}
$$

is the probability of $i$ consecutive unsuccessful transmission attempts before the successful delivery. The average energy consumption can then be approximated by

$$
\mathcal{J}=\frac{\mathcal{E}_{\text {data }}}{8 \cdot L} \text { (Joule per Bit). }
$$

Similarly, the expected transmission duration for an uplink data frame, $\mathcal{D}_{\text {data }}$, can be calculated by Eq. (17), and the average goodput is

$$
\mathcal{G}=\frac{8 \cdot L}{\mathcal{D}_{\text {data }}}(\mathrm{Mbps})
$$

\section{Evaluation Results And Discussion}

As specified in the IEEE 802.11 standard [2], the length of a MAC Service Data Unit (MSDU), which is a data unit conveyed from the higher Logic Link Control (LLC) sublayer to the MAC, can be up to 2304 octets (see Clause 6.2.1.1.2 in [2]). The maximum transmit power is limited to $200 \mathrm{~mW}$ (i.e., $23 \mathrm{dBm}$ ) [20] for the middle band of the $5 \mathrm{GHz}$ U-NII band, which is suitable for indoor environments. Clearly, the PA reaches the maximum power conversion efficiency, $\eta_{\max }$, when the output power level is $23 \mathrm{dBm}$. In this paper, we assume that all the MSDUs are 2304-octet long and transmitted without fragmentation. Furthermore, as mentioned in Section IV-A, we assume an exponential E-P curve for the $\mathrm{PA}$, where the power conversion efficiency is 0.02 when the output power level is $0 \mathrm{dBm}\left(\eta_{0}=0.02\right)$. Table $\mathrm{V}$ summarizes the power characteristics we used to obtain the evaluation results, and Figs. 7(a) and (b) show the E-P curves of a low-efficiency PA $\left(\eta_{\max }=0.1\right)$ and a high-efficiency PA $\left(\eta_{\max }=0.5\right)$, respectively. Recall that SNR (in $\left.\mathrm{dB}\right)$ is equal to $\left[\mathcal{P}_{\text {out }}(\mathrm{dBm})-\right.$ path_loss $(\mathrm{dB})$ - noiselevel $\left.(\mathrm{dBm})\right]$.

In order to evaluate the energy-consumption performance of a transmission scheme quantitatively, we introduce a new
TABLE V

POWER CHARACTERISTICS USED FOR THE EVALUATION

\begin{tabular}{|l||l|}
\hline $\mathcal{P}_{\text {com }}$ & $500 \mathrm{~mW}$ \\
\hline $\mathcal{P}_{\text {rec }}$ & $50 \mathrm{~mW}$ \\
\hline $\mathcal{P}_{\text {out }}$ & $-19-23 \mathrm{dBm}$ \\
\hline noise_level & $-93 \mathrm{dBm}$ \\
\hline$\eta_{\text {max }}$ & 0.1 or 0.5 \\
\hline
\end{tabular}
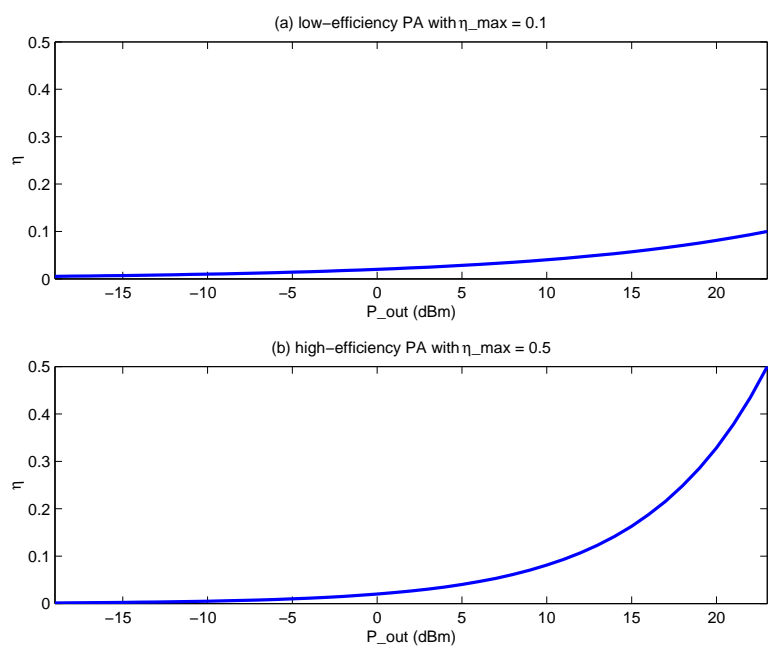

Fig. 7. E-P (efficiency vs. output power level) curves of $5 \mathrm{GHz}$ PA

measure called the energy consumption ratio. It is defined as the ratio of the energy consumption, when the referred scheme is used, to the energy consumption, when the adaptive PHY mode selection and TPC with 15 power levels are used (as shown in Fig. 8(c)). This measure presents how a particular scheme performs, in terms of the energy consumption, relative to the adaptive scheme of dynamic PHY mode selection 
and TPC with 15 power levels, which is found in our draft proposal [21] to the future IEEE 802.11h standard.

\section{A. Data Transmission with Minimum Energy Consumption}

First, we investigate the problem of selecting the proper combination of PHY mode and transmit power level to achieve energy-efficient uplink data transmissions for non-real-time applications (e.g., FTP-like services).

Fig. 8 shows the energy-consumption performance when there are 15 transmit power levels (with $3 \mathrm{dBm}$ steps) and when the PA presents a low power conversion efficiency $\left(\eta_{\max }=0.1\right)$. The optimal combinations of PHY mode and transmit power level, which achieve the most energy-efficient uplink data transmissions (i.e., $\mathcal{J}$ is minimized), under different path loss conditions are shown in Figs. 8(a) and (b), and the corresponding energy consumptions are shown in Fig. 8(c), respectively. For example, when the path loss is $100 \mathrm{~dB}$, this figure reads that, by using PHY mode 4 at the transmit power level of $17 \mathrm{dBm}$, the uplink data is transmitted with minimum energy consumption (about 0.08 mJoule per information bit).

Basically, we have two more observations from Fig. 8. First, when the path loss is large, the lower PHY modes are preferred since they are more robust and have better error performances. On the other hand, when the path loss is small, higher PHY modes are used to save energy since the duration of a single transmission attempt is shorter. Note that even with TPC, PHY mode 2 (BPSK modulation with rate-3/4 coding) is not part of the optimal selection due to its longer transmission time but only comparable error performance to PHY mode 3 (QPSK modulation with rate-1/2 coding) under most SNR conditions, which is consistent with a similar observation in [4], where the link adaptation idea was studied in order to maximize the system goodput. Second, a low transmit power level does not necessarily result in low energy consumption. This is because,
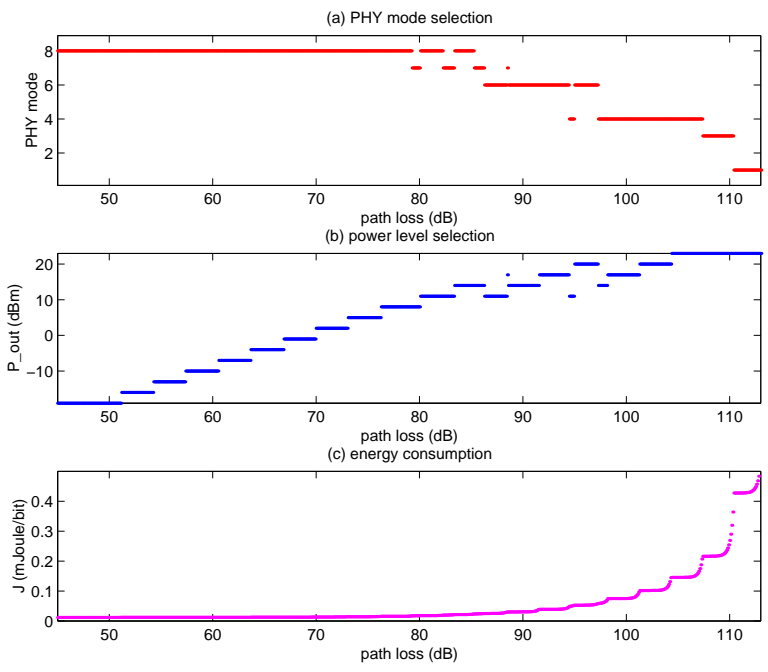

Fig. 8. 15 power levels with low-efficiency PA
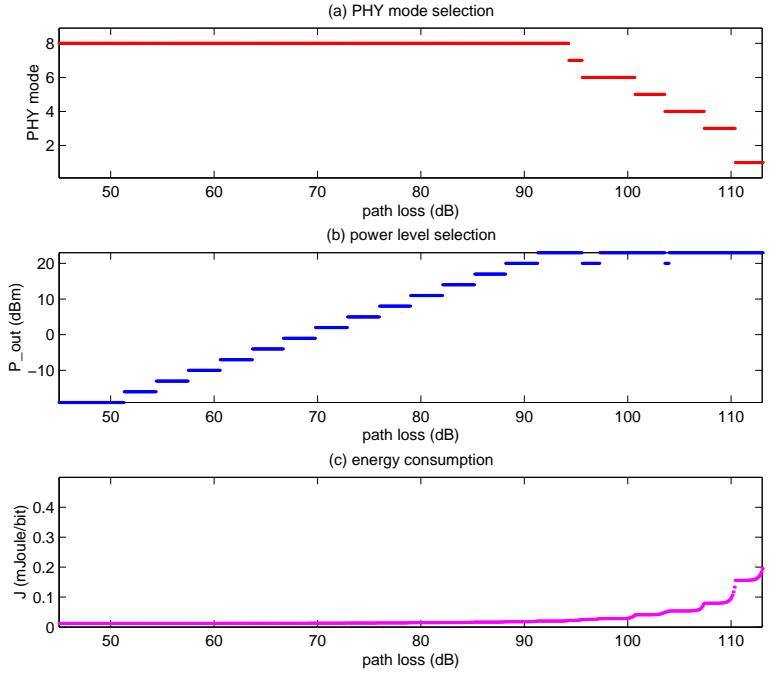

Fig. 9. 15 power levels with high-efficiency PA

for the same PHY mode, adopting a lower transmit power level may lead to less energy consumption in a single transmission attempt, but the consequent low SNR may cause more re-transmissions and greater total energy consumption.

The key idea is to select the best mode-power pair, rather than the PHY mode or the transmit power level itself, to minimize the energy consumption for each path loss value. Moreover, due to the discreteness of the available PHY modes (8) and transmit power levels (15), under a certain PA model, it is possible that the combination of a higher PHY mode with stronger transmit power results in lower energy consumption than the combination of a lower PHY mode with rather weaker transmit power. As shown in Fig. 8, when the path loss is about $80 \mathrm{~dB}$, PHY mode 7 is selected with the transmit power of $8 \mathrm{dBm}$, while for the path loss of slightly higher than 80 $\mathrm{dB}$, PHY mode 8 is used again, however, with a higher power level at $11 \mathrm{dBm}$. Similar switch-backs can also be observed at other path loss ranges in the figure. In comparison, when the transmit power level is fixed (see Figs. 10 and 11), the PHY mode selection becomes a non-increasing function of the path loss.

Fig. 9 shows the energy-consumption performance with high-efficiency PA $\left(\eta_{\max }=0.5\right)$. We can see that the two observations from Fig. 8 also hold, and additionally, the higher power levels are more likely to be selected due to the higher power conversion efficiency of the PA. Due to space limitations, we will present other evaluation results only for lowefficiency PA, but the same trends are actually found to hold for high-efficiency PA as well.

The energy-consumption performances with the transmit power level fixed at $15 \mathrm{dBm}$ (the nominal value of Agere ORiNOCO card) and $23 \mathrm{dBm}$ (the maximum allowed in the $5 \mathrm{GHz}$ middle band) are plotted in Figs. 10 and 11, respectively. As expected, we observe from Figs. 10(c) and 11(c) 

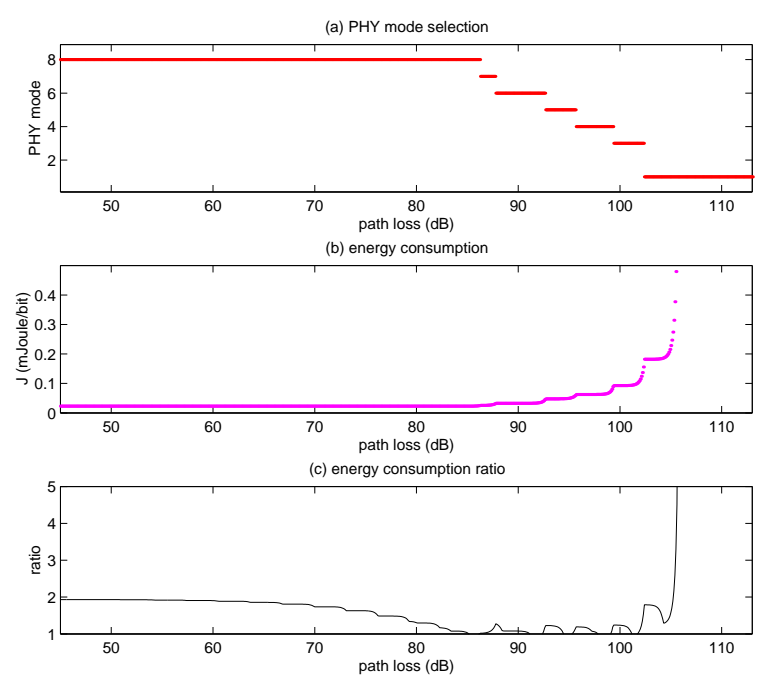

Fig. 10. Fixed power level at $15 \mathrm{dBm}$ with low-efficiency PA
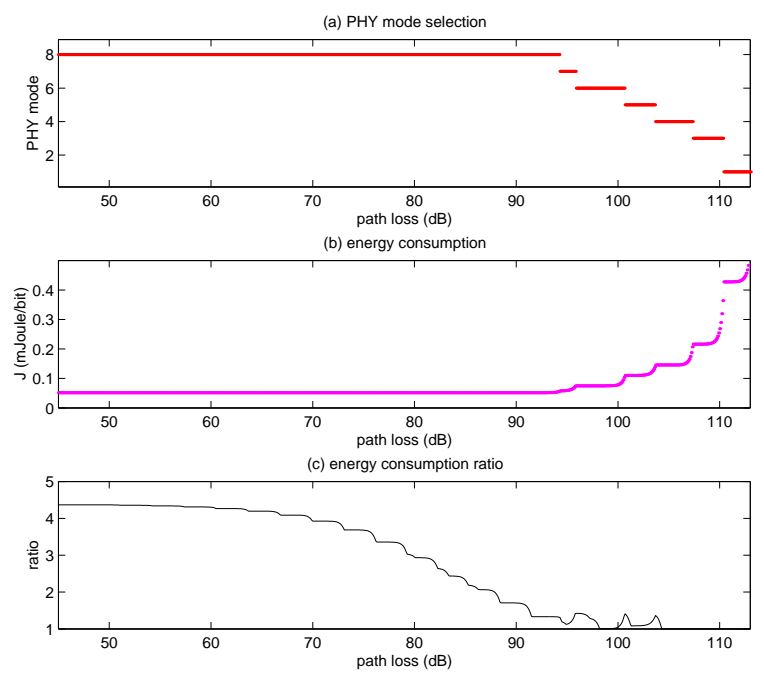

Fig. 11. Fixed power level at $23 \mathrm{dBm}$ with low-efficiency PA

that the schemes with fixed power levels consume more energy in general. Note that the larger the energy consumption ratio, the less energy-efficient. In Fig. 10, energy consumptions close to the optimum - corresponding to the energy consumption ratio one - can only be observed at the path loss range between $85 \mathrm{~dB}$ and $100 \mathrm{~dB}$, where the power level of $15 \mathrm{dBm}$ is part of, or close to, the optimal selection (see Fig. 8(b)). When the path loss is smaller than $80 \mathrm{~dB}$, the scheme consumes more energy because the frames are transmitted using a higher power level than necessary over a relatively short distance. When the path loss is larger than 105 $\mathrm{dB}$, the energy consumption goes up drastically (to infinity), meaning that even with the most robust PHY mode, the power level of $15 \mathrm{dBm}$ is still not high enough to combat the high path loss, and thus transmission never succeeds. On the other hand, if the fixed power level is increased to the maximum,
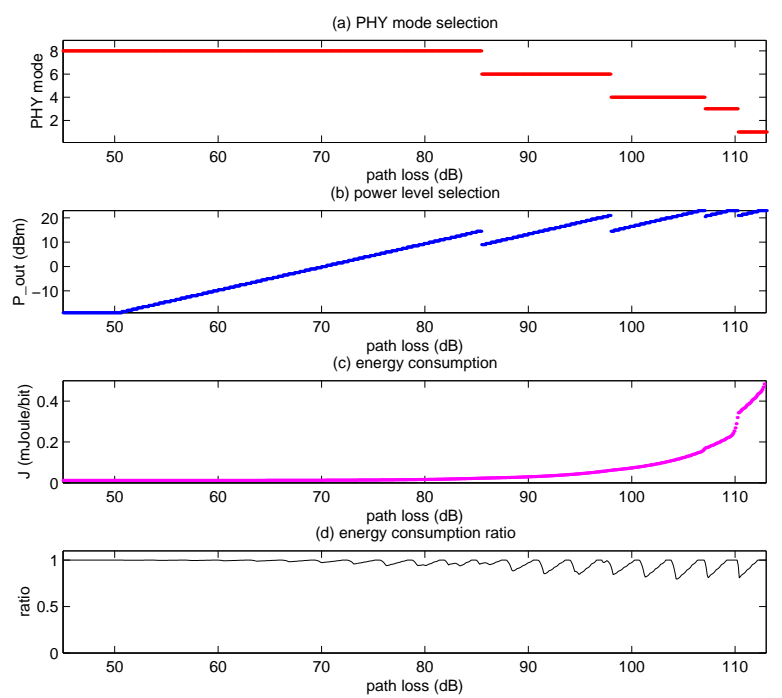

Fig. 12. 85 power levels with low-efficiency PA

i.e., $23 \mathrm{dBm}$, such a scheme works fine under high path loss conditions, as shown in Fig. 11. However, significantly more energy is consumed at the low path loss range, as an undesirable side effect. Based on the above observations, we draw the following conclusion: by simply adjusting the PHY mode while fixing the transmit power level, we will inevitably suffer either limited operating (path loss) range or much higher energy consumption.

Finally, we increase the number of transmit power levels from 15 to 85 (with $0.5 \mathrm{dBm}$ steps) and show the energyconsumption performance in Fig. 12. Compared to Fig. 8, we observe smoother PHY mode transitions and power level transitions due to the finer power levels. However, the results in Fig. 12(d) suggest that the energy gain may not be significant.

\section{B. Energy-Efficient Data Transmission with Goodput Con- straint}

Now, we consider the problem of selecting the optimal combination of PHY mode and transmit power level to achieve energy-efficient uplink data transmissions with additional goodput constraint (e.g., streaming-like services). The objective is to meet the minimum goodput requirement while saving as much energy as possible. Obviously, different results are expected from those in Section V-A.

Fig. 13 shows the results when 15 transmit power levels are used and the target goodput is set to $35 \mathrm{Mbps}$. We have three observations. First, when the path loss is smaller than $86 \mathrm{~dB}$, the 35 Mbps goodput target can be achieved with minimum energy consumption. The rationale is that there is no conflict between the energy-saving requirement and the goodput requirement when the station is close to the PC. Second, when the path loss is between $86 \mathrm{~dB}$ and $95 \mathrm{~dB}$, more energy than the minimum is required to achieve the target goodput. We can 

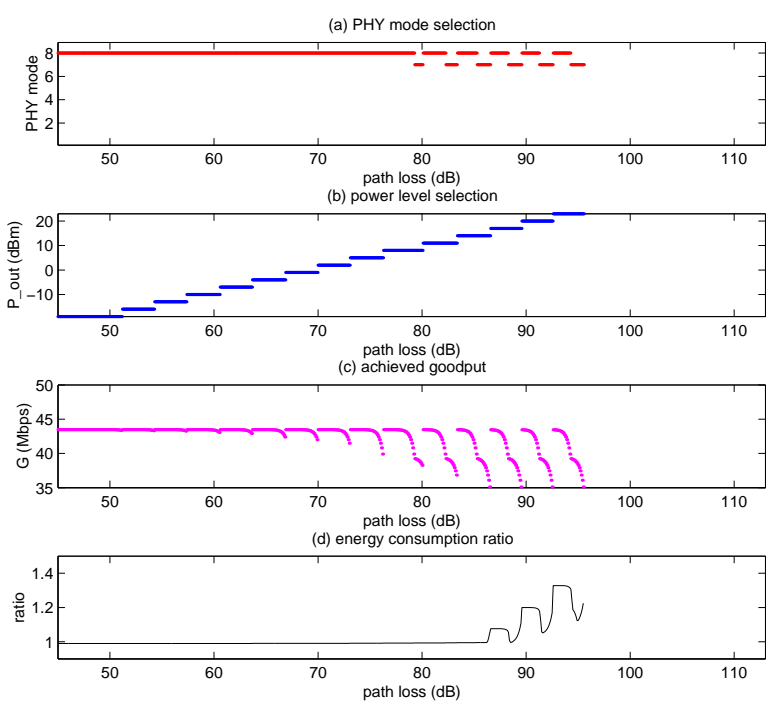

Fig. 13. 15 power levels with target goodput of $35 \mathrm{Mbps}$
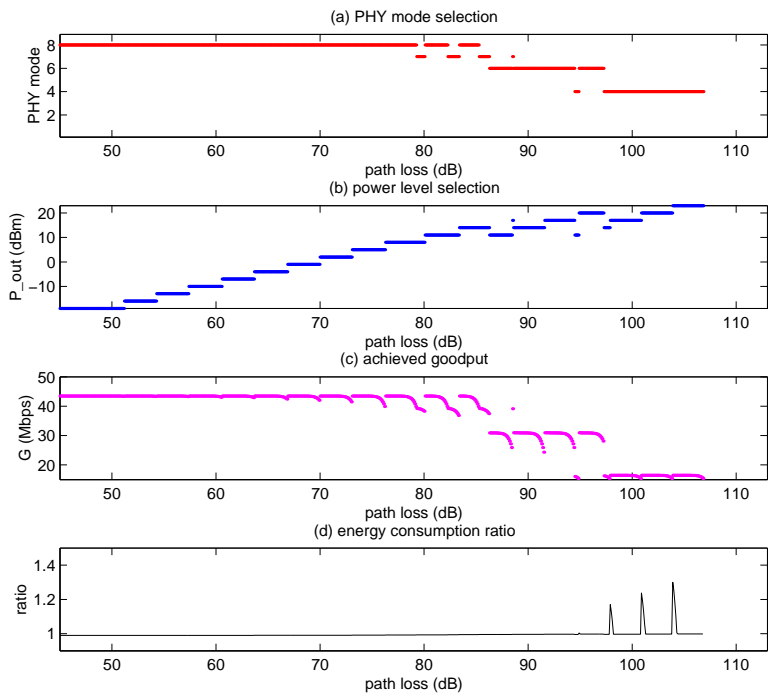

Fig. 14. 15 power levels with target goodput of $15 \mathrm{Mbps}$

see that PHY modes 7 and 8 are used at this path loss range, although they are not part of the optimal selections from a pure energy saving point of view (see Fig. 8(a)). This is because PHY modes 7 and 8 are the only two modes that may be able to achieve goodput equal to, or higher than, $35 \mathrm{Mbps}$. Consequently, higher power levels have to be selected at this path loss range, such that the error performances of PHY modes 7 and 8 can be improved enough to achieve the target goodput. Third, when path loss is larger than $95 \mathrm{~dB}$, the $35 \mathrm{Mbps}$ goodput target cannot be achieved due to limitation of the maximum transmit power level. Similar trends are observed for the target goodput of $15 \mathrm{Mbps}$, as shown in Fig. 14.

An alternative way to determine the mode-power pair to meet the target goodput requirement and save energy is as follows. The maximum transmit power is first assumed in determining the proper PHY mode to meet the target goodput. Once the PHY mode is selected, the transmit power is then reduced as much as possible while still meeting the target goodput. One problem with such a scheme is that it only gives a sub-optimal pair of PHY mode and transmit power level, because it divides a two-dimensional optimization problem into two one-dimensional problems without proper decoupling process.

\section{IMPLEMENTATION ISSUES}

In order to realize the proposed idea of adaptive PHY mode selection with TPC for uplink data transmissions, a wireless station has to estimate the path loss between itself and the PC. We developed a simple and novel scheme [21] for this purpose, and we are proposing it to be part of the upcoming IEEE $802.11 \mathrm{~h}$ standard, which is an extension to the current 802.11 MAC and 801.11a PHY. The main idea is to convey the transmit power level information in the SERVICE field of the PPDU.

As shown in Fig. 6, the DATA field of an IEEE 802.11a PPDU contains the 16-bit SERVICE field. The standard bit assignment of the SERVICE field is shown in Fig. 15. Bits $0-6$ are set to zero and are used to initialize the descrambler at the receiver. The remaining 9 bits $(7-15)$ of the SERVICE field are reserved for future use. As defined in the IEEE 802.11 standard [2], TXPWR_LEVEL is one of the TXVECTOR parameters, which are passed from MAC to $\mathrm{PHY}$, in order to initiate a MAC frame transmission. Other parameters in the TXVECTOR include the transmission rate (i.e., PHY mode) and the frame length. Currently, TXPWR_LEVEL is defined from 1 to 8, where the mapping between a TXPWR_LEVEL value and the actual power is implementation-dependent.

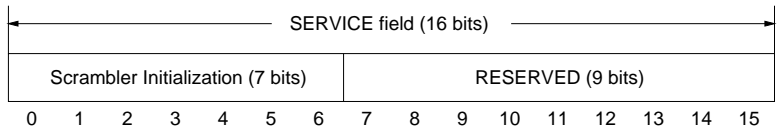

Fig. 15. Standard SERVICE field bit assignment

We have proposed to redefine TXPWR_LEVEL from 1 to 15, and standardize the mapping between a TXPWR_LEVEL value and the actual power level (in $\mathrm{dBm}$ ) so that the receiver of a PPDU can identify the transmit power level (in $\mathrm{dBm}$ ) of the received PPDU. The 15 transmit power levels are from $19 \mathrm{dBm}$ to $23 \mathrm{dBm}$ with $3 \mathrm{dBm}$ steps in the middle band of the $5 \mathrm{GHz}$ U-NII band as was used in Fig. 8. Fig. 16 illustrates our proposal to revise the SERVICE field bit assignment, which would use bits $7-10$ to convey the TXPWR_LEVEL information. We have also proposed to add one parameter, which is TXPWR_LEVEL extracted from the SERVICE field in the PPDU being received, into the RXVECTOR. Note that an RXVECTOR is passed from PHY to MAC via the PHYRXSTART.request(RXVECTOR) service primitive when the 
PHY starts conveying the received frame bit stream to the MAC.

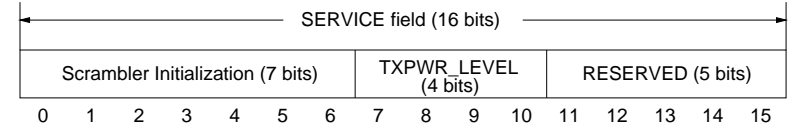

Fig. 16. Revised SERVICE field bit assignment

With the above proposed changes, it becomes possible for a wireless station to estimate the path loss between itself and the PC easily. That is, with the knowledge of the received signal strength (in $\mathrm{dBm}$ ) via the Receive Signal Strength Indicator (RSSI) as well as the transmit power level (in $\mathrm{dBm}$ ) via TXPWR_LEVEL found in the received frame's (e.g., CF-Ack or CF-Poll) SERVICE field, a wireless station can calculate the path loss (in $\mathrm{dB}$ ) by doing the simple subtraction. Note that RSSI is one of the RXVECTOR parameters, which is measured by the physical layer and indicates the energy observed at the antenna used to receive the current PPDU. Basically, the path loss calculated in this manner can be used by the wireless station to determine the best transmission strategy for its next uplink frame. For example, one can look up Fig. 8 to select the optimal combination of PHY mode and transmit power level for an FTP service, while referring to Fig. 13 for a data streaming service with the target goodput of $35 \mathrm{Mbps}$. This approach is reasonable since with 802.11 WLANs, the same frequency channel is used for all transmissions in a time-division duplex manner, and hence, the channel characteristics in terms of path loss for both directions between two stations are likely to be similar.

\section{CONCLUSION}

In this paper, we investigate and demonstrate the energy savings achieved for uplink data transmissions under the PCF via transmit power control (TPC) and PHY rate adaptation in IEEE 802.11a WLANs. Since we do not have access to the energy-consumption characteristics of the 802.11a-compliant products currently available in the market, we make some reasonable assumptions based on the power characteristics of two 802.11b-compliant WLAN devices, and analyze the energy consumption for uplink data transmissions in an IEEE 802.11a WLAN under the PCF. Evaluation results suggest that, by combining TPC with adaptive PHY mode selection, considerable energy savings can be achieved compared to those schemes that only adapt the PHY mode but fix the transmit power level. We also present a novel, simple, and effective way to calculate the path loss and realize our approach. This new scheme is being proposed to be part of the upcoming $802.11 \mathrm{~h}$ standard, which is currently being developed by the IEEE 802.11 Task Group H (TGh).

\section{ACKNOWLEDGMENT}

The authors would like to thank Dave Bryan and Javier del Prado at Philips Research USA for their reviews and comments on the earlier version of this paper.

\section{REFERENCES}

[1] Ramjee Prasad and Tero Ojanpera, "An Overview of CDMA Evolution toward Wideband CDMA," IEEE Communications Surveys, vol. 1, no. 1, pp. 2-29, Fourth Quarter 1998.

[2] IEEE 802.11, Part 11: Wireless LAN Medium Access Control (MAC) and Physical Layer (PHY) Specifications, Standard, IEEE, Aug. 1999.

[3] IEEE 802.11a, Part 11: Wireless LAN Medium Access Control (MAC) and Physical Layer (PHY) Specifications: High-speed Physical Layer in the $5 \mathrm{GHz}$ Band, Supplement to IEEE 802.11 Standard, Sep. 1999.

[4] Daji Qiao and Sunghyun Choi, "Goodput Enhancement of IEEE 802.11a Wireless LAN via Link Adaptation," in Proc. IEEE ICC'01, Helsinki, Finland, Jun. 2001.

[5] Steven D. Gray and Venkatesh Vadde, "Throughput and Loss Packet Performance of DCF with Variable Tranmit Power," IEEE 802.1101/227, May 2001.

[6] Sunghyun Choi, "PCF vs. DCF: Limitations and Trends," IEEE 802.1101/154, Jan. 2001.

[7] IEEE 802.11h/D1.0, Part 11: Wireless LAN Medium Access Control (MAC) and Physical Layer (PHY) Specifications: Spectrum and Transmit Power Management extensions in the $5 \mathrm{GHz}$ band in Europe, Draft Supplement to IEEE 802.11 Standard-1999 Edition, Draft 1.0, Jul 2001.

[8] Tajana Simunic, Luca Benini, Peter Glynn, and Giovanni De Micheli, "Dynamic Power Management for Portable Systems," in Proc. ACM MobiCom'00, Boston, MA, Aug. 2000, pp. 11-19.

[9] Mark Stemm, Paul Gauthier, Daishi Harada, and Randy H. Katz, "Reducing Power Consumption of Network Interfaces in Hand-Held Devices," in Proc. 3rd International Workshop on Mobile Multimedia Communications, Princeton, NJ, Sep. 1996.

[10] Paul Lettieri, Christina Fragouli, and Mani B. Srivastava, "Low Power Error Control for Wireless Links," in Proc. ACM MobiCom'97, Budapest, Hungary, 1997, pp. 139-150.

[11] J. G. Proakis, Digital Communications, 3rd ed., McGraw Hill, New York, NY, 1995.

[12] M. B. Pursley and D. J. Taipale, "Error Probabilities for SpreadSpectrum Packet Radio with Convolutional Codes and Viterbi Decoding," IEEE Transactions on Communications, vol. COM-35, no. 1, pp. 1-12, Jan. 1987.

[13] D. Haccoun and G. Begin, "High-Rate Punctured Convolutional Codes for Viterbi and Sequential Decoding," IEEE Transactions on Communications, vol. 37, no. 11, pp. 1113-1125, Nov. 1989.

[14] Agere Systems, User's Guide for ORiNOCO PC Card, Sep. 2000.

[15] Intersil Americas Inc., Prism II 11Mbps Wireless Local Area Network PC Card, Apr. 2001.

[16] Intersil Americas Inc., $2.4 \mathrm{GHz}$ Power Amplifier and Detector, Mar. 2000.

[17] IEEE 802.11b, Part 11: Wireless LAN Medium Access Control (MAC) and Physical Layer (PHY) Specifications: High-speed Physical Layer Extension in the $2.4 \mathrm{GHz}$ Band, Supplement to IEEE 802.11 Standard, Sep. 1999.

[18] John F. Sevic, "Statistical Characterization of RF Power Amplifier Efficiency for CDMA Wireless Communication Systems," in Proc. Wireless Communications Coonference, Boulder, CO, Aug. 1997, pp. 110-113.

[19] M. Rofougaran, A. Rofougaran, C. Olgaard, and A. A. Abidi, "A $900 \mathrm{MHz}$ CMOS RF Power Amplifier with Programmable Output," in 1994 Symposium on VLSI Circuits Digest of Technical Papers, Jun. 1994, pp. 133-134.

[20] Bob O'Hara and A1 Petrick, The IEEE 802.11 Handbook: A Designer's Companion, Standards Information Network, IEEE Press, 1999.

[21] S. Choi, S. Gray, M. Kasslin, S. Mangold, A. Soomro, A. Myles, D. Skellern, and P. Ecclesine, "Transmitter Power Control (TPC) and Dynamic Frequency Selection (DFS) Joint Proposal for $802.11 \mathrm{~h}$ WLAN," IEEE 802.11-01/169r2, May 2001. 